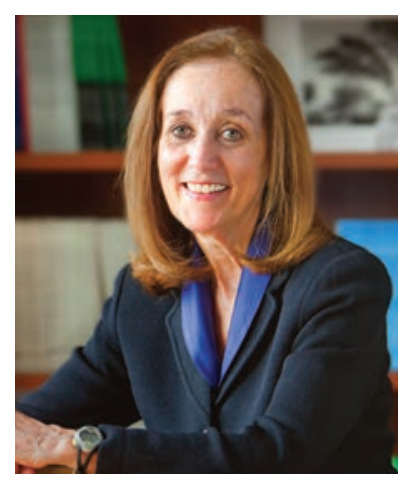

Mary S. McCabe, RN, MA

Mary S. McCabe, RN, MA, is Director of the Cancer Survivorship Program at Memorial Sloan-Kettering Cancer Center (MSKCC), where she is responsible for developing and implementing center-wide comprehensive programs for cancer survivors. She is also a faculty member in the Division of Medical Ethics at the Cornell Weill Medical College and Chair of the MSKCC Ethics Committee. A graduate of Trinity College, Emory University, and Catholic University, she held several positions at the $\mathrm{NCl}$ before joining MSKCC, including Assistant Director of the Division of Cancer Treatment and Diagnosis, Director of The Office of Clinical Research, and faculty in the Department of Bioethics at the NIH.

Ms. McCabe serves on many committees, including the Committee on Improving the Quality of Cancer Care at the IOM, the Survivorship Committee of ASCO, the Survivorship Steering Committee of the ACS, the NCCN Survivorship Panel, the $\mathrm{NCl}$ Clinical Trials and Translational Research Advisory Committee, and she is Chair of the ASCO Survivorship Committee. She is a member of ONS, ASCO, and the American Society for Bioethics and Humanities.

\section{Cancer Survivorship: We've Only Just Begun}

\author{
Mary S. McCabe, RN, MA
}

Editor's Note: To celebrate the finale of the Year of the Survivor in JNCCN, we are reprinting the below commentary that details the start of the NCCN Guidelines for Survivorship and thoughts on where the process of integrating survivorship care into oncology was just a few short years ago. For more thoughts on transitioning patients with cancer into survivorship care, please see the article on page 1681 of this issue.

Over the past decade, tremendous progress has been made in the early detection and effective treatment of many cancers, resulting in a growing population of cancer survivors in the United States that exceeds 13.7 million as of 2012. ${ }^{1}$ With the 5-year survival rate for individuals diagnosed with adult-onset cancers now almost $65 \%$, this gratifying number will certainly increase. As a result, survivorship is becoming a central component of oncology care as we increasingly take on the responsibility of optimizing health outcomes for cancer survivors. What began a decade ago as the interest of a few is becoming a focus of the majority.

The foundational prototype for cancer survivorship in this country is based on pediatric cancer care, because pediatric oncologists have been providing formal follow-up services to their patients for decades. This is due to a number of important factors: the high success rates in treating childhood cancers; the consistent practice of incorporating multidisciplinary services into care of the child beginning at diagnosis; and understanding of the long-term consequences of treatment exposures on developing organs and tissues. Their adult oncology colleagues are recent newcomers to this approach to follow-up care and are only now taking a critical look at how we can move from visits that focus on surveillance for recurrence to a strategy of comprehensive but tailored survivorship care.

\section{Getting Survivorship on the Oncology Radar Screen}

Initially, adult cancer survivorship was a patient-led movement joined by a few physician and nurse champions and organized into the National Coalition for Cancer Survivorship. Although this coalition was established in 1986, the voice of the organization has become strongest over the past decade, as it became influential in highlighting for the public, health care professionals, and policy makers the unmet needs of patients with cancer and their families after treatment ends. ${ }^{2}$

Other prominent national groups have also focused attention on cancer survivorship with the intention of making survivorship care a national priority. In 2004, the President's Cancer Panel released a report, Living Beyond Cancer, with recommendations for needed survivorship services based on interviews with survivors and family members. ${ }^{3}$ In the same year, the Lance Armstrong Foundation and the CDC published a National Action Plan for Cancer Survivorship, which included strategies that would advance cancer survivorship public health efforts around access to needed care and services, education and training, applied research, and the development of program infrastructure. ${ }^{4}$ In 2005, the Institue of Medicine (IOM) issued a report, From Cancer Patient to Cancer Survivor: Lost in Transition. ${ }^{5}$ This comprehensive publication has had, by far, the greatest impact and provided the most influential blueprint to date to guide survivorship program development in the United States. It has been significantly important to groups developing their own comprehensive survivorship agendas abroad.
The ideas and viewpoints expressed in this commentary are those of the author and do not necessarily represent any policy, position, or program of NCCN. 


\section{Progress to Date}

Accepting the challenge of the IOM report, cancer centers around the country were the first to begin formal survivorship programs and services; however, in the past few years, these programs have increasingly become established by community hospitals and private practices as well. This is an important evolution because these centers and practices are where most patients receive care.

Over the past decade, models of care have been developed to serve the needs of varying groups of survivors and implemented in ways that reflect the resources of the practice or facility. Formalizing care in such a way that includes surveillance for recurrence, assessment of long-term and late effects, cancer screening, health promotion recommendations, and communication among providers drives the critical change needed to make survivorship care a reality. ${ }^{6}$ However, in doing this, cancer centers and community programs alike have struggled to develop financially sustainable services in the absence of evidence-based guidelines for a standardized approach to care for specific survivor groups. These models are just the beginning effort to answer first-order questions that must be addressed to allow provision of quality survivorship care. These questions have significant implications for research funders, professional organizations, patient advocacy groups, and policy makers: What is the right care; when is the right time; and who is the right provider?

Fortunately, we are beginning to address these questions, and national organizations are increasingly extending their reach to include survivorship in their overall mission. The year 2011 marked a significant turning point for cancer survivorship nationally, as influential groups and organizations publicly made survivorship a priority. ASCO established a Survivorship Committee with a comprehensive agenda that includes evaluating models of survivorship care and developing guidance recommendations for follow-up with an initial focus on long-term and late effects.

That same year, the American Cancer Society established a Survivorship Steering Committee to advise their newly established National Cancer Survivorship Resource Center. The focus of this CDC-funded effort is on developing guidance for the followup care for individuals treated for melanoma, breast, prostate, head and neck, lung, colorectal, and gynecologic cancers using an exposure-based approach.

In 2012, NCCN formed a Survivorship Panel that is developing guidelines for the care of the cancer survivor, focusing initially on recommendations for immunizations, exercise, chronic pain, sleep disturbances, sexual issues, anxiety, depression, fatigue, and cognitive function. Each in their own way, these critically important organizations are setting out complementary agendas to fill the gaps in our knowledge about what constitutes quality posttreatment care so that the information tools will be readily available.

\section{Attention to Change}

However promising the work of these national groups is, other critical factors we must consider include reconfiguring the survivorship work force and redesigning oncology services. One important reason these changes are critical is that the need for oncology services is projected to increase at a rate that the oncologist work force cannot meet. ASCO has reported that by 2020 , partly because of the increasing numbers of elderly people, there will be a shortage of 2350 to 3800 oncologists. $^{7}$ Who will care for these cancer patients, many of whom will become cancer survivors?

Fortunately, the survivorship community has initiated models of care with nurse practitioners and physician assistants, and preliminary results regarding satisfaction among survivors and oncologists has been very positive. ${ }^{8,9}$ Additional evaluation of these novel care models will be important. An evaluation of cost along with clinical 
outcomes will allow for a broader reconfiguring of the well-established team approach to cancer care that has long been a tradition.

The second important change going forward involves rethinking the oncology services needed by survivors. A focus on developing a thoughtful risk-based approach to care is gaining support so that survivors receive appropriate oncology services while they are at risk for recurrence and late effects, but can transition to primary care providers when the risks are low. For this approach to be successful, concerns about the relationship between the oncology and primary care providers-in particular the transfer of knowledge-must be resolved using the Chronic Care Model already in place for other diseases as the prototype. ${ }^{10}$

\section{Challenges Going Forward}

One of the most important challenges going forward will be to thoughtfully incorporate the guidance that groups such as NCCN and ASCO are developing, acknowledging the currently limited scientific evidence. To do this in such a way that ultimately improves care and promotes the conduct of outcomes research requires the sustained commitment of these professional organizations and a community of researchers devoted to establishing evidence-based survivorship care.

A second challenge we face is assuring that, as our health care system changes (especially with the implementation of the Affordable Care Act), the needs of survivors are acknowledged and financial rewards are provided for coordinating care among specialists and generalists. A third challenge is the growing population of elderly cancer survivors as a unique and complicated constituency. The health problems of this group already have an impact on oncology care nationally and will require major attention over the next decade. The influence of a cancer diagnosis on preexisting comorbidities, life goals, and family supports will require additional attention by all involved.

\section{Conclusions}

Finally, the progress in cancer survivorship has been significant, with a real acceleration in interest and commitment nationally. Although challenges to be addressed and met certainly remain, the opportunities are great. We are well on our way to improving the health and quality of life of cancer survivors.

\section{References}

1. Cancer Treatment of Survivorship: Facts and Figures 2012-2013. American Cancer Society, 2012.

2. Clark EJ, Stoval EL, Leigh S, et al. Imperatives for quality cancercare: access, advocacy, action and accountability. National Coalition for Cancer Survivorship. Silver Spring, MD; 1996.

3. President's Cancer Panel. Living Beyond Cancer: Finding a new balance-President's Cancer Panel Report. Washington, DC: US Dept of Health and Human Services; 2004.

4. Centers for Disease Control and Prevention. A National Action Plan for Cancer Survivorship: Advancing Public Health Strategies. Washington, DC: US Dept of Health and Human Services; 2004.

5. Hewitt M, Greenfield S, Stovall E, eds. From Cancer Patient to Cancer Survivor: Lost in Transition. Washington, DC: Institute of Medicine and National Research Council; 2005.

6. Oeffinger K, McCabe MS. Models of delivering survivorship care. J Clin Oncol 2006;24:5117-5124.

7. Erikson C, Salsberg E, Forte G, et al. Future supply and demand for oncologists. J Oncol Pract 2007;3:79-86.

8. McCabe MS, Jacobs L. Survivorship care: models and programs. Semin Onc Nurs 2008;24:202-207.

9. Buswell LA, Ponte PR, Shulman LB. Provider practice models in ambulatory oncology practice: analysis of productivity, revenue, and provider and patient satisfaction. J Oncol Pract 2009;5:188-192.

10. Del Giudice ME, Grunfeld E, Harvey BJ, et al. Primary care physicians' views of routine follow-up care of cancer survivors. J Clin Oncol 2009;27:3338-3345. 